\title{
Development, Characterization and In Vitro Evaluation of Flurbiprofen Solid Dispersions using Polyethylene Glycols as Carrier
}

\author{
Bindu Yadav", Y. S. Tanwar \\ ${ }^{1}$ Department of Pharmaceutics, Bhupal Nobles' College of Pharmacy, Udaipur, Rajasthan, India.
}

\section{ARTICLE INFO \\ Article history: \\ Received on: 01/10/2015 \\ Revised on: 05/01/2016 \\ Accepted on: 29/01/2016 \\ Available online: 30/04/2016}

Key words:

Solubility, FLB, Solid

dispersion, IR spectroscopy,

\begin{abstract}
The purpose of the present investigation was to increase the solubility and dissolution rate of flurbiprofen (FLB) by the preparation of its solid dispersion with polyethylene glycol 4000 and 6000 as carriers using solvent evaporation method (SM) and kneading method (KM). Drug polymer interactions were investigated using Fourier transform infrared spectroscopy (FTIR), differential scanning calorimetry (DSC), x-ray diffraction (XRD). The prepared solid dispersions were characterized by IR spectroscopy, which suggests no interaction of drug with carriers. XRD and DSC study indicates reduction in drug crystallinity. Drug solubility was more and also the dissolution was rapid for Flurbiprofen solid dispersions compared to pure drug.
\end{abstract}

\section{INTRODUCTION}

Oral bioavailability of a drug depends on its solubility and/or dissolution rate, and dissolution may be the ratedetermining step for the onset of therapeutic activity. Therefore, poorly aqueous soluble drugs are usually characterized by a low bioavailability due to less absorption, which is a major concern of pharmaceutical industries worldwide (Hasnain and Nayak, 2012). Techniques that have commonly been used to improve dissolution and bioavailability of poorly water-soluble drugs, in general include micronization, the use of surfactant, and the formation of solid dispersions (Sammour et al., 2006). Solid dispersion is one of the techniques that can potentially enhance the dissolution rate of hydrophobic drugs with pharmacologically inert, polymeric materials (Shin and Kim, 2003). The term 'solid dispersion' has been utilized to describe a family of dosage forms whereby the drug is dispersed in a biologically inert matrix, usually with a view to enhancing oral bioavailability (Craig, 2002). The improvement of solubility and dissolution rate of drugs from solid dispersions is based on mainly three different mechanisms includes; increased wettability of drug due to direct contact with hydrophilic carrier, the reduction in particle size results increased surface area, and the conversion of crystalline state to more soluble amorphous state (De Waard et al., 2008).

* Corresponding Author

E-mail: binduyadav24@gmail.com
Propionic acid derivatives, like Flurbiprofen (FLB), are considered to be the first line drugs in the symptomatic treatment of rheumatoid arthritis, osteoarthritis, and ankylosing spondylitis. They are safe and effective analgesic and by inhibiting the prostaglandin synthesis they can interrupt the normal paracrine signaling necessary for the elaboration of inflammatory response (Brooks and Day, 1991; Qiu and Bae, 2006). Flurbiprofen has been found to be one of the most potent members of a series of phenylalkanoic acids in various animal species in antiinflammatory, analgesic and antipyretic tests (Van Miert and Van Duin, 1977). It is highly effective and safe in the treatment of rheumatoid arthritis. Flurbiprofen also causes a dose dependent inhibition of collagen induced platelet aggregation in platelet rich plasma from human, rats and rabbit's in-vitro (Nishizawa et al., 1973). Area under the plasma drug concentration versus time curve increases with increasing dose administration (Kaiser et al., 1986). It has a short elimination half- life of $3.9 \mathrm{hr}$. and is slightly soluble in water (Verma and Murthy, 1997). Since the rate of Flurbiprofen absorption is controlled by the release of drug from its dosage form into the gastrointestinal tract. The major drawback in the therapeutic use and efficacy of Flurbiprofen as oral dosage form is its very low aqueous solubility because of its hydrophobic nature. Poor aqueous solubility and slow dissolution rate of the drug leads to low oral bioavailability consequently irreproducible. Therefore a better oral formulation was developed by increasing the water solubility of drug. 
The literature survey reveals that the solubility of poorly water soluble drugs can be enhanced by solid dispersion using polyethylene glycols (Perng et al., 1998; Khan, and Zhu., 1998; Dubois, and Ford, 1985; Betageri, and Makarla, 1995). Hence in the present study, solubility of Flurbiprofen (FLB) will be improved by solid dispersion methods' using different water soluble carries.

\section{MATERIAL AND METHODS}

\section{Materials}

Flurbiprofen (FLB) was received as a gift sample from Sun Pharma (Ahemadnagar), PEG 6000 and PEG 4000 was purchased from $\mathrm{CDH}$, New Delhi. Double distilled water is used throughout the study and other chemicals were of analytical or pharmaceutical grade.

\section{Development of Solid Dispersions (SDs)}

\section{Physical Mixture}

The physical mixtures (1:1) were prepared by weighing the calculated amounts of Flurbiprofen and carriers. Then mix them in a glass mortar by triturating. The resultant physical mixtures were passed through 60-mesh sieve and stored in desiccators until used for further studies.

\section{Kneading Method (KM)}

The calculated amounts of drug and polymer were weighed and mixed in the different ratios 1:1, 1:3, 1:5. The mixtures were kneaded with small volume of water for $30 \mathrm{~min}$ in a glass mortar to produce a homogeneous dispersion. The paste formed was dried in oven at $45{ }^{\circ} \mathrm{C}$ under vacuum until the solid dispersion was dry. The dispersions after drying were passed through 60 \# sieve. The granules obtained were stored in desiccators until used for further studies (Table no 1 and 2).

Table 1: Composition of Various Solid Dispersions of FLB with PEG 4000.

\begin{tabular}{cccc}
\hline $\begin{array}{c}\text { Formulation } \\
\text { Code }\end{array}$ & Carrier & $\begin{array}{c}\text { Drug : } \\
\text { Carrier } \\
\text { Ratio }\end{array}$ & Method \\
\hline PM $_{144}$ & PEG 4000 & $1: 1$ & Physical Mixture \\
KM$_{154}$ & PEG 4000 & $1: 5$ & Kneading Method \\
SM $_{154}$ & PEG 4000 & $1: 5$ & Solvent Evaporation Method \\
\hline
\end{tabular}

Table 2: Composition of Various Solid Dispersions of FLB with PEG 6000.

\begin{tabular}{cccc}
$\begin{array}{c}\text { Formulation } \\
\text { Code }\end{array}$ & Carrier & $\begin{array}{c}\text { Drug : } \\
\text { Carrier } \\
\text { Ratio }\end{array}$ & Method \\
\hline PM $_{116}$ & PEG 6000 & $1: 1$ & Physical Mixture \\
KM $_{156}$ & PEG 6000 & $1: 5$ & Kneading Method \\
SM $_{156}$ & PEG 6000 & $1: 5$ & Solvent Evaporation Method \\
\hline
\end{tabular}

\section{Solvent Evaporation Method (SM)}

The required amount of drug and polymer was dissolved in acetone in 1:1 proportions and transferred to a petri dish; the solvent was allowed to evaporate at room temperature, until a major portion of the solvent used was volatilized. Further for complete removal of solvent it is dried in oven at $45{ }^{\circ} \mathrm{C}$ until dryness. The dried mass was pulverized, passed through a 60 \# sieve and stored in desiccators at room temperature until further evaluation (Table 1 and 2).

\section{Solubility Study}

Solubility determinations were performed in triplicate according to the method of Higuchi and Connors (Higuchi and Connors, 1965). In brief, an excess amount (equivalent to $40 \mathrm{mg}$ of drug) of samples (pure Flurbiprofen, physical mixtures and Flurbiprofen solid dispersions) were added to $25 \mathrm{ml}$ distilled water in a stopper conical flask and the mixtures were rotated for $24 \mathrm{hrs}$ in a rotary flask shaker $\left(37 \pm 0.5^{\circ} \mathrm{C}\right)$.

The mixtures were filtered through a $0.45 \mu \mathrm{m}$ membrane filter. The filtrate was suitably diluted and analyzed spectrophotometrically at the wavelength of $247 \mathrm{~nm}$ using a UVVIS spectrophotometer (Varian USA. Model: Carry 50).

\section{EVALUATION OF FLUBIPOFEN SOLID DISPERSION SYSTEMS}

\section{Drug-Content}

SDs was weighed accurately (equivalent to $10 \mathrm{mg}$ of flurbiprofen) and dissolved in $10 \mathrm{ml}$ of methanol. The drug content was analyzed at $247 \mathrm{~nm}$ spectrophotometrically after suitable dilutions. (Perkin Elmer, USA). Each sample was analyzed in triplicate.

\section{In Vitro Dissolution}

The dissolution study was carried out using USP XXII apparatus type II (Electrolab TDT-06T). The dissolution medium was $900 \mathrm{ml}$ of distilled water kept at $37 \pm 0.5{ }^{\circ} \mathrm{C}$, with rotational speed of $50 \mathrm{rpm} .5 \mathrm{ml}$ of test fluid were withdrawn at specified time intervals and filtered through a membrane filter $(0.45 \mu)$. The same volume of fresh medium was added to the dissolution medium. The samples were analyzed spectrophotometrically at $247 \mathrm{~nm}$. Each preparation was tested in triplicate and the mean values were calculated.

\section{Statistical Comparisons}

The dissolution profiles were compared using two parameters DP30 (percentage of Flurbiprofen dissolved at $30 \mathrm{~min}$ ) and DP60 (percentage of Flurbiprofen dissolved at $60 \mathrm{~min}$ ). The comparisons were made between the methods and carriers by analysis of variance (ANOVA). The dissolution release kinetics and the results of best-fit model among the preparations were also compared.

\section{FTIR Study}

The Infrared spectra (IR) of Flurbiprofen and some selected preparations were obtained using FTIR (Perkin Elmer 1600 Series). The IR spectroscopy was carried out by $\mathrm{KBr}$ pellet method and scanned at wavelengths $4000 \mathrm{~cm}^{-1}$ to 400 $\mathrm{cm}^{-1}$. 


\section{Differential Scanning Calorimetry (DSC)}

The DSC thermograms were recorded using a differential scanning calorimeter (DSC 8000 Perkin Elmer, USA). Approximately 3-5 mg of each samples was accurately heated from 20 to $150{ }^{\circ} \mathrm{C}$ at a scanning rate of $10^{\circ} / \mathrm{min}$., purge gas nitrogen $20 \mathrm{ml} / \mathrm{min}$.

\section{X-Ray Diffractrometry (XRD)}

Powder X-ray diffraction patterns were recorded using X-ray diffractometer (Phillips X-Ray Diffractometer PW 1710) under the following conditions. $\mathrm{Ni}$ - filter $\mathrm{CU}-\mathrm{K} \alpha$ radiation, $40 \mathrm{KV}$ voltages: $30 \mathrm{~mA}$ current, scan speed $2^{0} \mathrm{~min}$ in turns of $2 \theta$.

\section{RESULT AND DISCUSSION}

\section{Drug Content}

The drug content in all the tested combinations was found to be in the range of $95.91 \pm 0.70$ to $99.89 \pm 0.04 \%$ for Physical mixture and solid dispersion respectively. Table no 3 indicates the applications of the present method for the preparation of SDs with high content uniformity.

Table 3: \% Drug Content, Solubility Data of Physical Mixture \& Solid Dispersions.

\begin{tabular}{lcccc}
\hline $\begin{array}{c}\text { Formulations } \\
\text { Code }\end{array}$ & \multicolumn{2}{c}{ PEG 4000 } & \multicolumn{2}{c}{ PEG 6000 } \\
\cline { 2 - 5 } & $\begin{array}{c}\text { Percentage } \\
\text { Drug Content }\end{array}$ & $\begin{array}{c}\text { Solubility in } \\
\text { Water(mg/ml) }\end{array}$ & $\begin{array}{c}\text { Percentage } \\
\text { Drug Content }\end{array}$ & $\begin{array}{c}\text { Solubility in } \\
\text { Water(mg/ml) }\end{array}$ \\
\hline PM 1:1 & $98.04 \pm 0.12$ & $0.117 \pm 0.09$ & $96.80 \pm 0.35$ & $0.139 \pm 0.01$ \\
KM 1:5 & $98.12 \pm 0.62$ & $0.279 \pm 0.12$ & $99.06 \pm 0.42$ & $0.285 \pm 0.12$ \\
SM 1:5 & $99.78 \pm 0.42$ & $0.296 \pm 0.07$ & $98.62 \pm 0.61$ & $0.349 \pm 0.04$ \\
\hline
\end{tabular}

\section{Saturation Solubility of FLB Solid Dispersions}

Among all the PMs and SDs the carrier PEG 6000 containing formulations showed enhanced solubility results (Fig.1). The 1:5 ratios SD showed high solubility may be due to higher proportion of hydrophilic carriers present in SDs (Table no 3). On applying t-test there is no significant difference found in physical mixtures and drug solubility. But there is significant difference present in solubility of drug and SDs prepared both by kneading method and solvent evaporation method (1:5). These results are in accordance with the established formation of soluble complex between water-soluble polymeric carriers and poorly soluble drug (Mura et al., 1996, Zerrouk et al., 2002).

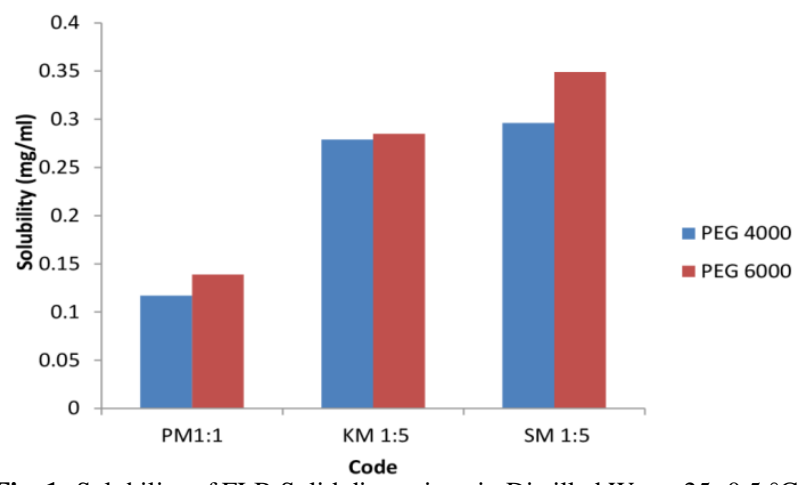

Fig. 1: Solubility of FLB Solid dispersions in Distilled Water $25 \pm 0.5{ }^{\circ} \mathrm{C}$

\section{FTIR Spectroscopy Studies}

FTIR spectroscopy was used to characterize the possible interactions between drug and carrier in the solid state. The IR spectra of SDs were compared with the standard spectrum of Flurbiprofen and PEG alone. The FTIR spectrum of pure FLB demonstrated peaks at $1701 \mathrm{~cm}^{-1}\left(\mathrm{C}=\mathrm{O}\right.$ stretching), $1217 \mathrm{~cm}^{-1}(\mathrm{C}-\mathrm{F}$ stretching), $2980.03 \mathrm{~cm}^{-1}$ (C-H stretching), $3031.89 \mathrm{~cm}^{-1}(\mathrm{C}-\mathrm{H}$ stretching) and $3647.14 \mathrm{~cm}^{-1}$ (O-H stretching). The FTIR spectrum of PM shows the characteristic peaks of drugs but with small decrease in intensity of peaks. FTIR spectra of solid dispersions $\mathrm{KM}_{154}, \mathrm{SM}_{154}, \mathrm{KM}_{156}$ and $\mathrm{SM}_{156}$ (Fig 2 and 3) show no substantial shifting of the position of the functional groups. The peaks are only broadened, indicating no major interaction between Flurbiprofen and hydrophilic carriers (Ahuja et al., 2007).

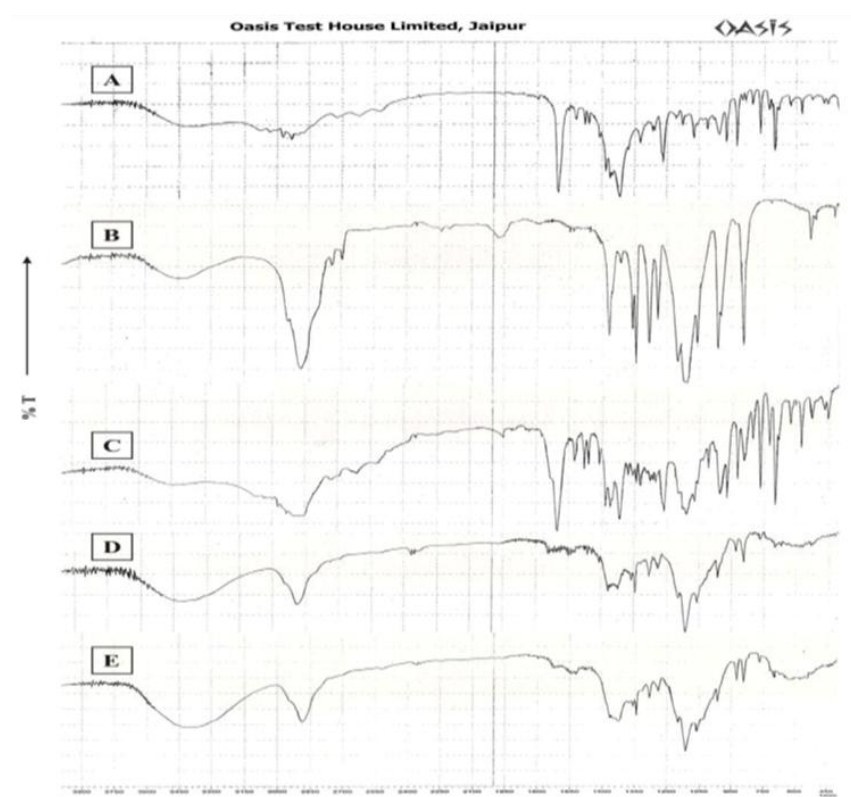

Fig. 2: FTIR Spectra of: (A) FLB,( B)PEG 4000, (C)PM, (D) KM 1:5, (E) SM 1:5.

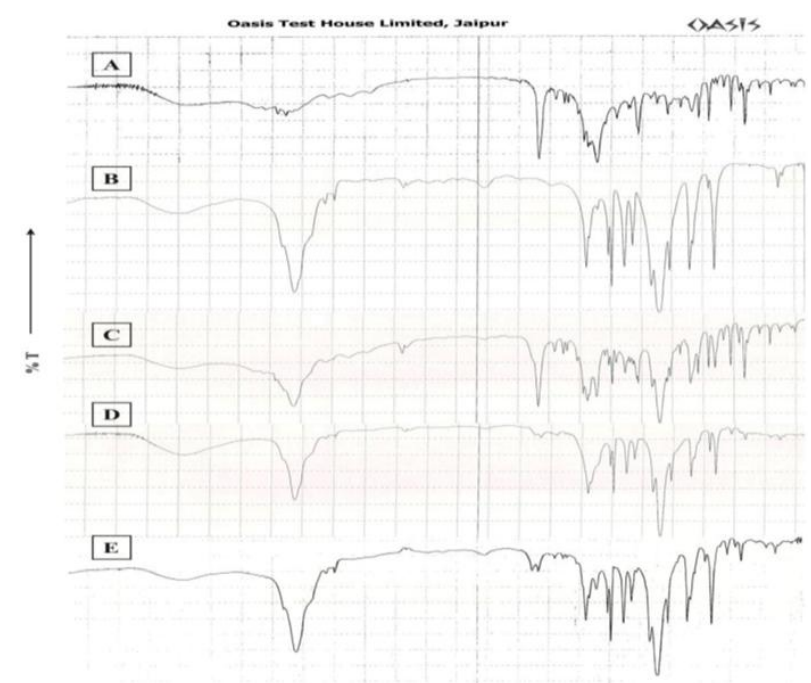

Fig. 3: FTIR Spectra of : (A) FLB, (B) PEG 6000, (C) PM, (D) KM 1:5, (E) SM 1:5. 


\section{Differential Scanning Calorimetry (DSC)}

The thermograms of the pure drug (FLB), polymer, SDs and PMs are illustrated in fig.4 and 5. DSC thermogram of Flurbiprofen (Figure 4a) show an endothermic peak at $116.80{ }^{\circ} \mathrm{C}$ corresponding to the melting point of Flurbiprofen $(\Delta \mathrm{H} 112.17$ $\mathrm{J} / \mathrm{g}$ ), indicating it's crystalline nature (Soliman et al., 2005). The thermal behavior of PEG 4000 and PEG 6000 exhibited a sharp but slightly broad endothermic peak at $58.58{ }^{\circ} \mathrm{C}, 61.94{ }^{\circ} \mathrm{C}$ respectively.

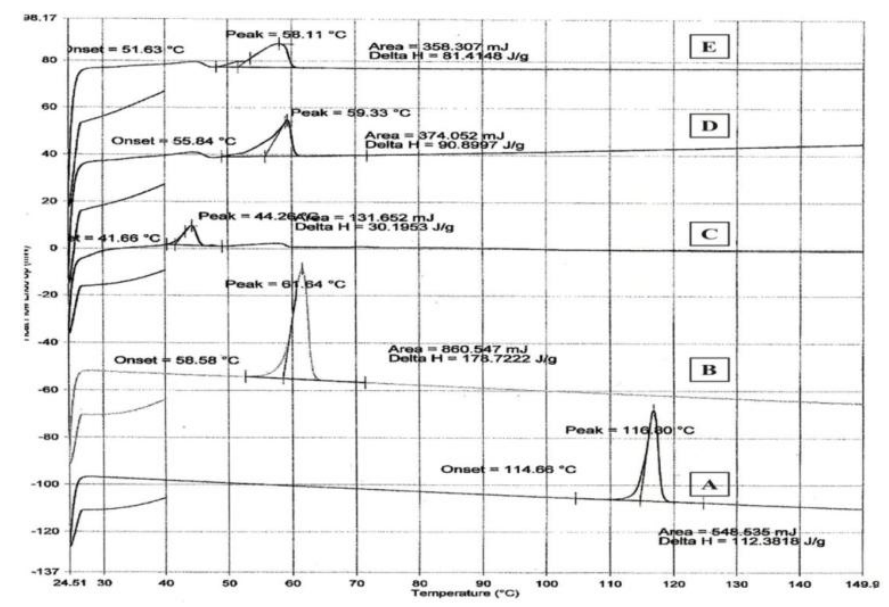

1) Heat rom $20.00^{\circ} \mathrm{C}$ to $180.00^{\circ} \mathrm{C}$ at $10.00^{\circ} \mathrm{C} / \mathrm{Cm}$

Fig. 4: DSC thermograms of: (A) FLB (B) PEG 4000 (C) PM (D) KM 1:5 (E) SM 1:5.

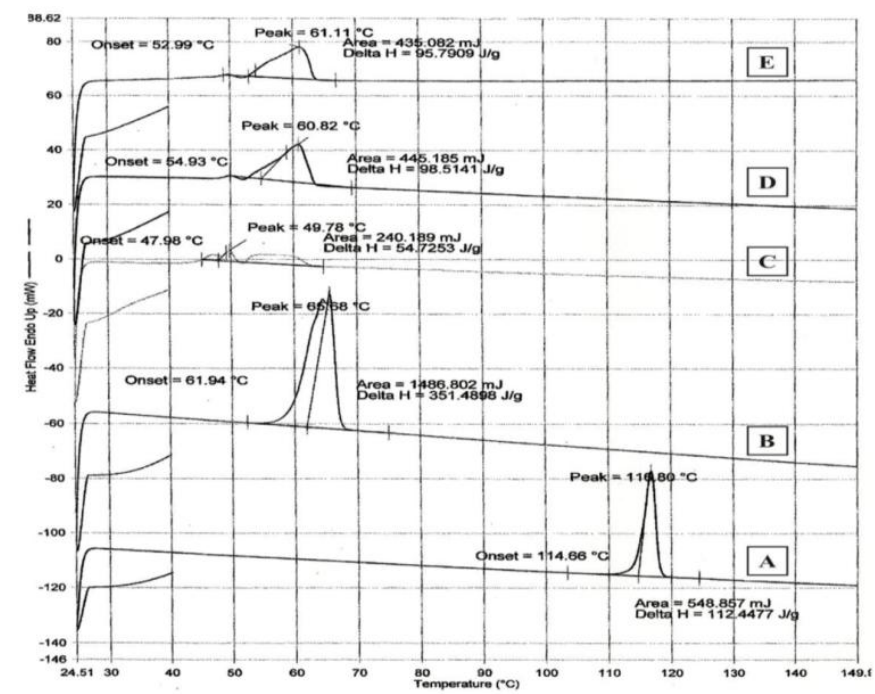

1) Heat from $20.00^{\circ} \mathrm{C}$ to $180.00^{\circ} \mathrm{C}$ at $10.00^{*} \mathrm{C} / \mathrm{m}$

Fig. 5: DSC thermograms of (A) FLB (B) PEG 6000 (C) PM (D) KM 1:5 (E) SM 1:5.

DSC curves of physical mixtures of carriers demonstrated a broadening or almost complete disappearance of FLB peaks together with a shift to a lower temperature than pure FLB and enthalpy of fusion of FLB was decreased. The DSC curves of PEG 4000 and PEG 6000 sharp FLB peaks were lost in all the SDs. In $\mathrm{KM}_{154}$ and $\mathrm{KM}_{156}$ PEG 4000, PEG 6000 the onset was $59.33{ }^{\circ} \mathrm{C}$, and $60.82{ }^{\circ} \mathrm{C}$, respectively. In $\mathrm{SM}_{154}$ and $\mathrm{SM}_{156} \mathrm{PEG}$
4000, PEG 6000 the broad reduced endotherm was observed with onset was $58.11{ }^{\circ} \mathrm{C}$ and $61.11{ }^{\circ} \mathrm{C}$ respectively. Complete disappearance of the flurbiprofen melting peak observed in both PM and SD was attributable to complete miscibility of the drug in the melted carrier. The enthalpy of drug melting in solid dispersion was gradually decreased as compared to the drug. This phenomenon could be attributed to the amorphous form of the drug in solid dispersion (Damian et al., 2000, Lin CW and Cham, 1996).

\section{X-Ray Diffractrometry (XRD)}

The powder XRD patterns of various Flurbiprofen, PEG 4000, PEG 6000 and its solid dispersions were compared in fig. 6 and 7. The diffraction pattern of pure drug showed, it's highly crystalline nature as indicated by numerous distinctive peaks at $2 \theta$ were $6.97,10.60,15.65,16.17,20.41,21.22,23.49$. PEG 4000 and 6000 exhibited two high intensity peaks at 19.07, 23.26 .

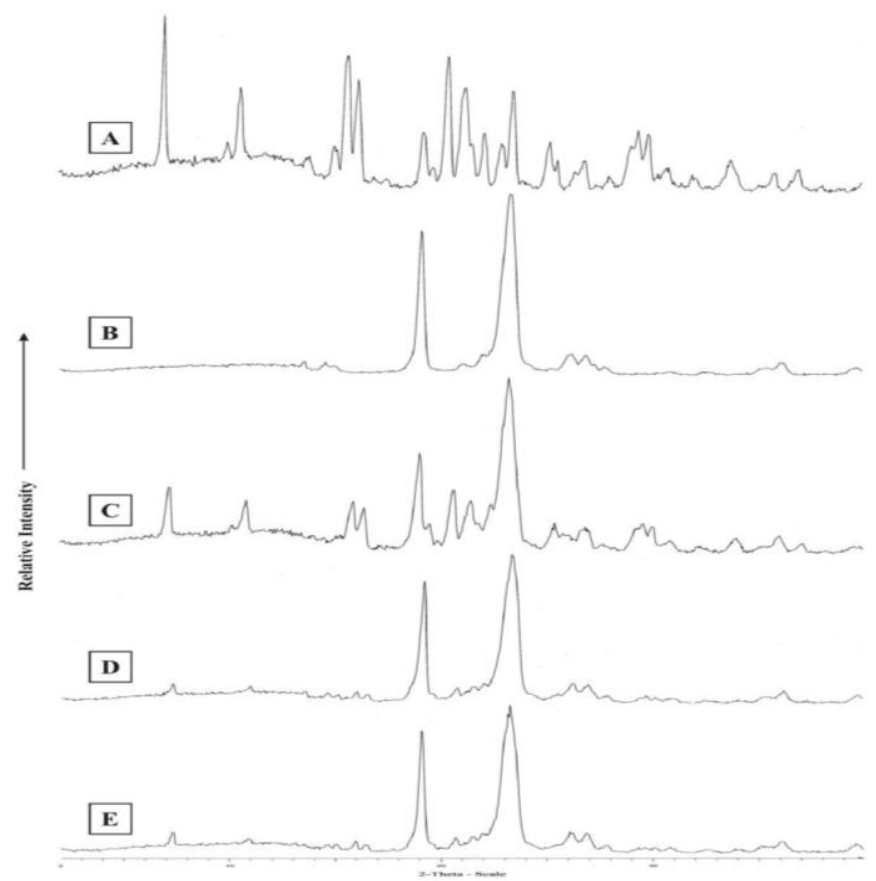

Fig. 6 : X-ray Diffraction Patterns of Pure FLB (A), PEG 4000 (B), Physical Mixture (PM) C) and SD using PEG 4000 Combination Carrier - KM (D) SM (E)

Diffraction patterns of SDs display the characteristic peaks of both FLB and polymers but the intensity and number of drug peaks are reduced suggesting there is a decrease in drug crystallinity (Parmar et al., 2011). Thus less intense peak in SD as compared to pure drug indicates amorphous nature results in higher solubility and dissolution rate as compared to pure drug (Reza et al., 2014). Thus with increasing polymer concentration, the crystallinity of the drug reduces further conversion to completely amorphous state, this explains that greater miscibility of FLB in the polymer at (1:5) ratio and deceased crystalline peaks which are obtained. 


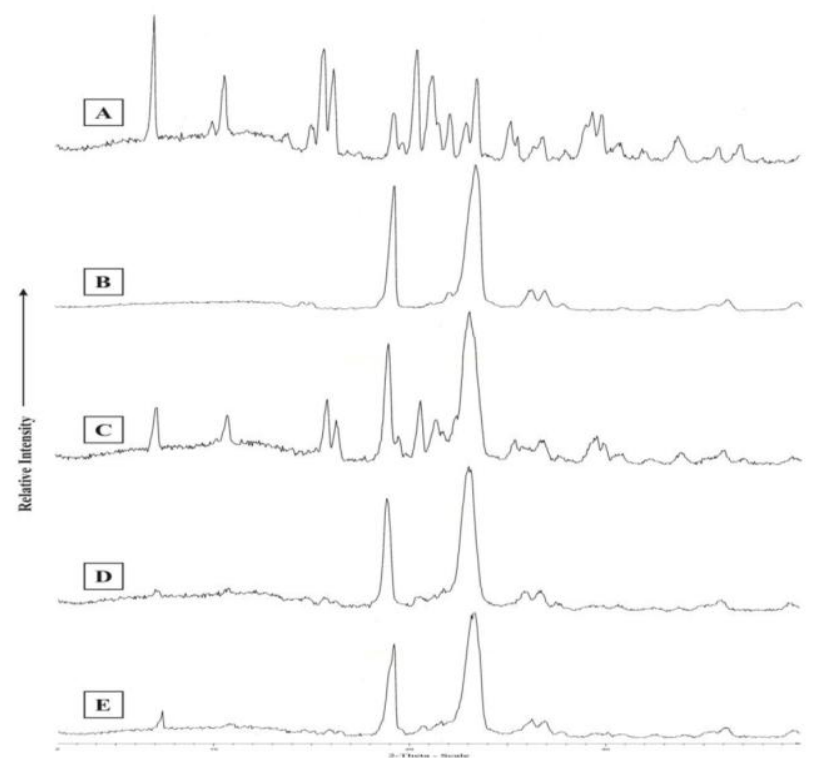

Fig. 7: X-Ray Diffraction Patterns of Pure FLB (A), PEG 6000 (B), Physical Mixture (PM) C), and SD using PEG 6000 Combination Carrier- KM (D), SM (E).

\section{Dissolution Study}

The solid dispersions of Flurbiprofen with PEG 4000 and 6000 showed improved dissolution when compared with physical mixtures and pure drug (Fig 8 and 9). The trend observed for percent dissolution of Flurbiprofen from solid dispersions containing PEG 6000 and 4000 prepared by physical mixing, kneading method and solvent evaporation methods, was an increase in dissolution rate with an increase in PEG percentage.

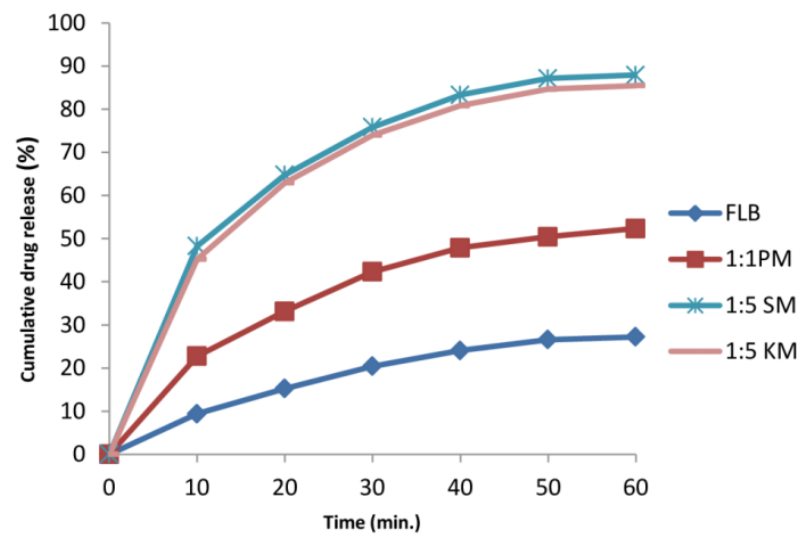

Fig. 8: Dissolution Profile of FLB from Physical Mixtures and SDs with PEG 4000 (Distilled water)

The dissolution of pure drug was only about $27.23 \%$ in distilled water at the end of $60 \mathrm{~min}$. The release of drug from formulation of PEG 4000 containing SD (SM 1:5) were $80.54 \%$ and PEG 6000 containing SD (SM 1:5) reached a maximum of about $85.45 \%$ at the end of $60 \mathrm{~min}$. in distilled water. Results in table no 4 suggest that all solid dispersions increased dissolution efficiency as compared to that of FLB in powder form. On applying ANOVA there is significant difference found in $\% \mathrm{DE}$ and $\%$ DR of all the formulations. As the concentration of polymer increased the DE increased significantly $(\mathrm{P}<0.05)$ due to enhanced wetting of FLB particles in the presence of hydrophilic groups of polymers (1:5). Tuckey's test revealed that all pairs differ significantly and highest mean was found for 1:5 SM. SDs prepared by solvent evaporation method showed a higher dissolution of FLB than those prepared by physical mixture and kneading, while SDs prepared by kneading technique showed a rather variable dissolution. This may be attributed to the fact that SDs prepared by solvent evaporation method result in a more uniform dispersion of drug in the hydrophilic carrier matrix as compared to those prepared by the kneading method (Dhingra et al., 2010).

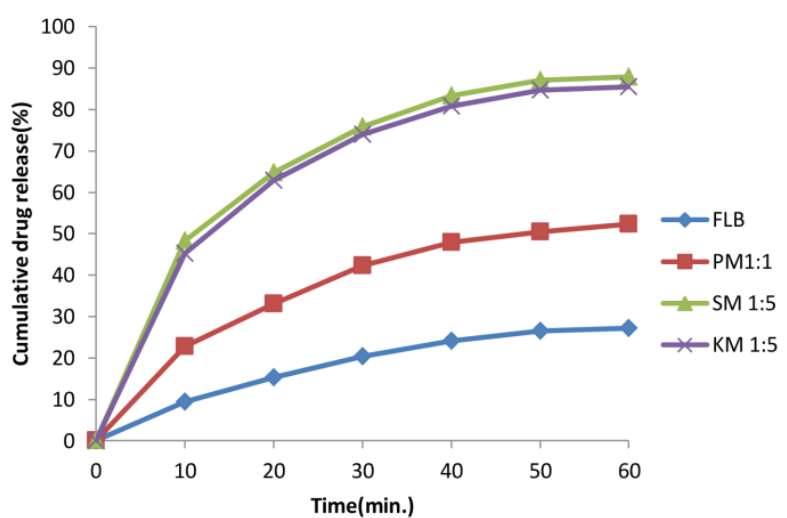

Fig. 9: Dissolution Profile of FLB from Physical Mixtures and SDs with PEG 6000 (Distilled water).

Table 4: \% Dissolution Efficiency and \% Drug Released of SDs in Distilled Water.

\begin{tabular}{cccc}
\hline $\begin{array}{c}\text { Formulation } \\
\text { Code }\end{array}$ & $\begin{array}{c}\text { \%Drug } \\
\text { Dissolved } \\
\left(\mathbf{Q}_{\mathbf{6 0}}\right)\end{array}$ & $\begin{array}{c}\text { Dissolution } \\
\text { Efficiency } \\
\left(\boldsymbol{\%} \mathbf{D E}_{\mathbf{3 0}}\right)\end{array}$ & $\begin{array}{c}\text { Dissolution } \\
\text { Efficiency } \\
\left(\boldsymbol{\%} \mathbf{D E}_{\mathbf{6 0}}\right)\end{array}$ \\
\hline FLB & $27.23 \pm 0.73$ & $11.63 \pm 0.35$ & $18.23 \pm 0.25$ \\
$\mathbf{P M}_{\mathbf{1 1 4}}$ & $51.73 \pm 0.33$ & $25.59 \pm 0.56$ & $36.37 \pm 0.25$ \\
$\mathbf{P M}_{\mathbf{1 1 6}}$ & $52.34 \pm 0.64$ & $25.79 \pm 0.48$ & $37.31 \pm 0.27$ \\
$\mathbf{S M}_{\mathbf{1 5 4}}$ & $83.60 \pm 0.48$ & $46.05 \pm 0.23$ & $63.08 \pm 0.27$ \\
$\mathbf{S M}_{\mathbf{1 5 6}}$ & $87.92 \pm 0.15$ & $50.32 \pm 0.97$ & $67.21 \pm 0.33$ \\
$\mathbf{K M}_{\mathbf{1 5 4}}$ & $80.54 \pm 0.22$ & $47.89 \pm 0.26$ & $62.85 \pm 0.17$ \\
$\mathbf{K M}_{\mathbf{1 5 6}}$ & $85.85 \pm 0.11$ & $48.39 \pm 0.17$ & $65.06 \pm 0.22$ \\
\hline
\end{tabular}

Physical mixture of PEG also improves the dissolution profile of Flurbiprofen due to its hydrophilic nature but not such an extent as by kneading method and solvent evaporation method. As the proportion of PEG increased, Flurbiprofen dissolution rates increased up to an extent after that decreased; may be due to the localization of higher amounts of carrier itself. The improvement of dissolution may be due to reducing particle size of Flurbiprofen and hence improving drug wettability and significantly improved dissolution.

Table 5 lists the regression parameters obtained after fitting various release kinetics models to in vitro dissolution data. The goodness of fit for various models investigated for binary systems ranks in the order of Korsemeyer-Peppas> Higuchi > firstorder> zero-order. Results were based on the $r^{2}$ and residual sum of squares (SSR) values The Korsemeyer-Peppas model describes drug release kinetics in the most be fitting manner. 
Table 5: Kinetics of Drug Release from SDs.

\begin{tabular}{|c|c|c|c|c|c|c|c|c|c|}
\hline \multirow{2}{*}{$\begin{array}{c}\text { Formulation } \\
\text { Code }\end{array}$} & \multicolumn{2}{|c|}{ Zero-order } & \multicolumn{2}{|c|}{ First-order } & \multicolumn{2}{|c|}{ Higuchi model } & \multicolumn{2}{|c|}{ Korsmeyer-Peppas model } & \multirow[b]{2}{*}{ "n" value } \\
\hline & $\mathbf{R}^{2}$ & SSR & $\mathbf{R}^{2}$ & SSR & $\mathbf{R}^{2}$ & SSR & $\mathbf{R}^{2}$ & SSR & \\
\hline FLB & 0.9257 & 87 & 0.9475 & 55 & 0.9931 & 8 & 0.9918 & 8 & 0.6148 \\
\hline $\mathbf{P M}_{114}$ & 0.8505 & 549 & 0.9259 & 256 & 0.9952 & 19 & 0.9964 & 7 & .04401 \\
\hline $\mathbf{P M}_{116}$ & 0.8578 & 564 & 0.9275 & 244 & 0.9938 & 27 & 0.9897 & 22 & 0.4790 \\
\hline $\mathrm{SM}_{154}$ & 0.7756 & 2186 & 0.7526 & 329 & 0.9815 & 201 & 0.9849 & 58 & 0.3837 \\
\hline $\mathrm{SM}_{156}$ & 0.7380 & 2716 & 0.9594 & 348 & 0.9451 & 293 & 0.9870 & 43 & 0.3463 \\
\hline $\mathrm{KM}_{154}$ & 0.7882 & 1943 & 0.9464 & 341 & 0.9833 & 170 & 0.9843 & 62 & 0.3974 \\
\hline $\mathrm{KM}_{156}$ & 0.7511 & 2479 & 0.9519 & 363 & 0.9816 & 256 & 0.9825 & 56 & 0.3644 \\
\hline
\end{tabular}

$\mathrm{R}^{2}$ - Regression Coefficient $\quad$ SSR-Sum of Residuals Squares, n- Diffusion Coefficient

The value of diffusional exponent " $n$ " was obtained from the slopes of the fitted Korsemeyer-Peppas model (Aggarwal et al., 2010). The solid dispersion tablets tended to exhibit Fickian diffusional characteristics, as the corresponding values of $n$ were lower than the standard value from declaring Fickian release behavior, the results point out the prevalence of diffusional mechanistic phenomena.

\section{CONCLUSION}

Solid dispersion provides a simple and effective method of increasing solubility and oral bioavailability of poorly watersoluble drugs. The present study demonstrated the suitability of PEG 4000 and PEG 6000 as a carrier for the preparation of SDs of FLB. FTIR, XRD and DSC studies, offered an explanation of better dissolution rate from its SDs. The solubilization effect of PEG 6000 results in the reduction of aggregation of the drug particles, reduction of crystallinity, increased wettability and dispersibility and alteration of the surface properties of the drug particles. The Korsmeyer-Peppas model properly describes the dissolution data, possibly suggesting Fickian diffusion as the mechanism of drug release from SDs. Flurbiprofen-PEG 6000 combination provides a promising approach to enhance the solubility and dissolution rate of the drug.

\section{ACKNOWLEDGEMENTS}

The authors acknowledge the Sun Pharma Ahemadnagar, Mumbai India for providing me drug as a gift samples. The authors also thank to Department of Pharmaceutics, Bhupal Nobles' College of Pharmacy, Udaipur, Rajasthan, for providing facilities.

\section{REFERENCES}

Aggarwal S., Gupta GD., Chaudhary S. Solubility and Dissolution Enhancement of Poorly Aqueous Soluble Drug Atorvastatin Calcium using Modified Gum Karaya as Carrier: In vitro-In vivo evaluation. Int J Drug Del. 2012; 4: 341-365.

Ahuja N., Katare OP., Singh B. Studies on Dissolution Enhancement and Mathematical Modeling of Drug Release of a Poorly Water-Soluble Drug using Water Soluble Carriers. Eur J Pharm Biopharm. 2007; 65 (1): 26-38.

Betageri GV., Makarla KR. Enhancement of Dissolution of Glyburide by Solid Dispersion and Lyophilization Techniques, Int J Pharm. 1995; 126: 155-160.

Brooks P., Day R. Normal Anti-Inflammatory Drugs: Differences and Similarities. N Eng J Med. 1991; 324: 1716-25.
Craig D.Q.M. The mechanisms of Drug Release from Solid Dispersions in Water-Soluble Polymers. Int J Pharm. 2002; 231:131-144.

Damian FN., Blaton L., Naesens J., Balzarini and Kinget R., Augustijns P., Van den Mooter G. Physicochemical characterization of solid dispersions of the antiviral agent UC-781 with polyethylene glycol 6000 and gelucire 44/14. Eur J Pharm Sci. 2000; 10 (4): 311-322.

De Waard H., Hinrichs WLJ., and Frijlink HW. A novel bottom-up process to produce drug nanocrystals: controlled crystallization during freeze-drying. J Control Release. 2008; 128:179-183.

Dhingra D., Bhandari A., Sharma RB., Gupta R., Gupta S. Enhancement of Dissolution Rate of Slightly Soluble Drug Clomiphene Citrate by Solid Dispersion. Int J Pharm Tech Res. 2010; 2(3):1691-1697.

Dubois JL., Ford JL. Similarities in the Release Rates of Different Drugs from Polyethylene Glycol 6000 Solid Dispersions. J Pharm Pharmacol. 1985; 37: 494-495.

Fukuhara A., Imai T., Inoue K., Otagiri M. Effect of Oral Multiple-Dose Administration of Anti-inflammatory Flurbiprofen Chimera Drug on Gastric Lesion, other Toxicities and Disposition Kinetics. Biol Pharm Bull. 1995; 18:140-147.

Hasnain MS., Nayak AK. Solubility And Dissolution Enhancement of Ibuprofen by Solid Dispersion Technique Using PEG 6000-PVP K 30 Combination Carrier. Bulgarain J Sci Edu. 2012; 21:118 131.

Higuchi T., Connors KA. Phase Solubility Techniques. Adv Anal Chem Instrum. 1965; 4: 117-212.

Kaiser DG., Brooks CD., Lomen PL. Pharmacokinetics of flurbiprofen. The American Journal of Medicine. 1986; 80: 10-15.

Khan GM., Zhu JB. Preparation, Characterization, and Dissolution Studies of Ibuprofen Solid Dispersions using Polyethylene Glycol (PEG), Talc, and PEG-talc as Dispersion Carriers. Drug Dev Ind Pharm. 1998; 24: 455-462.

Lin CW., Cham TM. Effect of Particle Size on the Available Surface Area of Nifedipine from Nifedipine-Polyethylene Glycol 6000 Solid Dispersions. Int J Pharm. 1996; 127:261-272.

Mura P., Manderioli A., Bramanti G., Ceccarelli L. Properties of solid dispersions of naproxen in various polyethylene glycols. Drug Dev Ind Pharm. 1996; 22: 909-916.

Nishizawa EE., Wynalda DJ., Suydam DE., Molony BA. Flurbiprofen, a New Potent Inhibitor of Platelet Aggregation Thrombosis Res. 1973; 3: 577-588.

Parmar KR., Shah SR., Sheth NR. Studies in Dissolution Enhancement of Ezetimibe by Solid Dispersions in Combination with a Surface Adsorbent. Dissol Tech. 2011; 55-61.

Perng CY., Kearney AS., Patel K., Palepu NR., Zuber G. Investigation of Formulation Approaches to Improve the Dissolution of SB-210661, a Poorly Water Soluble 5-Lipoxygenase Inhibitor. Int J Pharm. 1998; 176:31-38.

Qiu L., Bae Y. Polymer Architecture and Drug Delivery. Pharm Res. 2006; 23: 1-30.

Sammour OA., Zidan AS., Hammad MA., Megrab NA. Formulation and Optimization of Mouth Dissolve Tablets Containing Rofecoxib Solid Dispersion. AAPS Pharm Sci Tech. 2006; 7(2): 162-69.

Shin SC., Kim J. Physicochemical Characterization of Solid Dispersion of Furosemide with TPGS. Int J Pharm. 2003; 251:79-84.

Siahi-Shadbad Mohammad Reza., Ghanbarzadeh Saeed., Barzegar-Jalali1Mohammad., Valizadeh Hadi., Taherpoor Alireza., 
Mohammadi Ghobad., Barzegar-Jalali Azim., Khosro Adibkia. Development and Characterization of Solid Dispersion for Dissolution Improvement of Furosemide by Cogrinding Method. Adv Pharm Bull. 2014; 4(4): 391-399.

Soliman MS., Khan MA. Preparation and in vitro characterization of a semi-solid dispersion of flurbiprofen with Gelucire44/14 and Labrasol. Pharmazie. 2005, 60(4), pp 288-293.

Van Miert AS., Van Duin CT. The Antipyretic Effect of Flurbiprofen. Eur J Pharm. 1977; 44: 197-204.

Verma PRP., Murthy TEGK. Transdermal flurbiprofen delivery using HPMC matrices: Design, in vitro and in vivo evaluation. Drug Dev Ind Pharm. 1997; 23(7): 633-8.
Zerrouk N., Chemtob C., Arnaud P., Toscani S., Dugue J. In vitro and in vivo evaluation of carbamazepine-PEG 6000 solid dispersions. Int J Pharm. 2002; 225: 49-62.

\section{How to cite this article:}

Yadav B., Tanwar YS. Development, Characterization and In Vitro Evaluation of Flurbiprofen Solid Dispersions using Polyethylene Glycols as Carrier. J App Pharm Sci, 2016; 6 (04): 060-066. 